\title{
A spatial vaccination strategy to reduce the risk of vaccine-resistant variants
}

\section{Xiyun Zhang}

Jinan University https://orcid.org/0000-0002-7694-234X

\section{Gabriela Lobinska}

Weizmann Institute of Science

\section{Michal Feldman}

Tel Aviv University

\section{Eddie Dekel}

Tel Aviv University

\section{Martin Nowak}

Harvard University

\section{Yitzhak Pilpel}

Weizmann Institute of Science

\section{Yonatan Pauzner}

Hayovel Elementary School

\section{Baruch Barzel}

Bar-llan University https://orcid.org/0000-0001-8862-4384

Ady Pauzner ( $\square$ pauzner@gmail.com )

Tel Aviv University https://orcid.org/0000-0003-2722-6686

\section{Article}

Keywords: COVID-19, spatial vaccination strategy, vaccine-resistant variants

Posted Date: October 15th, 2021

DOI: https://doi.org/10.21203/rs.3.rs-969637/v1

License: (c) (i) This work is licensed under a Creative Commons Attribution 4.0 International License.

Read Full License 
A spatial vaccination strategy to reduce the risk of vaccine-resistant variants

\author{
Xiyun Zhang ${ }^{1}$, Gabriela Lobinska², Michal Feldman ${ }^{3}$, Eddie Dekel ${ }^{4}$, Martin A Nowak ${ }^{5}$, \\ Yitzhak Pilpel ${ }^{2}$, Yonatan Pauzner ${ }^{6}$, Baruch Barzel ${ }^{7}$, Ady Pauzner ${ }^{8, *}$
}

${ }^{1}$ Department of Physics, Jinan University, Guangzhou, China

2 Department of Molecular Genetics, Weizmann Institute of Science, Israel

${ }^{3}$ School of Computer Science and Center for Combating Pandemics, Tel Aviv University, Israel

${ }^{4}$ Department of Economics, Northwestern University, IL, USA and School of Economics, Tel Aviv University, Israel

${ }^{5}$ Department of Mathematics and Department of Organismic and Evolutionary Biology, Harvard University, MA, USA

6 Hayovel Elementary School, Tel-Aviv, Israel

7 Department of Mathematics, Bar-Ilan University, Israel

8 School of Economics and Center for Combating Pandemics, Tel Aviv University, Israel

* Corresponding author. Email: pauzner@tauex.tau.ac.il

\begin{abstract}
:
The process of vaccinating the world population against COVID-19 is expected to take well over a year to complete. As vaccination progresses and population immunity increases, a counteracting relaxation of social distancing measures is observed. The result will be a prolonged period of high disease prevalence combined with a fitness advantage for vaccine-resistant variants, implying a considerably increased probability that a resistant variant will spread in the population. In this paper we propose a spatial vaccination strategy that has the potential to dramatically reduce this risk. Instead of spreading the vaccination effort equally throughout a country, distinct geographic regions of the country are sequentially vaccinated, quickly bringing each to effective herd immunity. Regions with high vaccination rates will then have low infection rates and vice versa. Since people primarily interact with others in their own region, spatial vaccination will reduce the number of encounters between infected people (the source of mutations) and vaccinated people (who facilitate the spread of vaccine-resistant strains). Thus with proper logistic preparations, a spatial vaccination campaign could be highly effective in reducing the global risk of vaccine-resistant variants.
\end{abstract}




\section{Introduction}

Whereas the rapid development of effective vaccines against COVID-19 represents a remarkable scientific achievement, manufacturing and logistic constraints are currently limiting vaccination rates. The process of global vaccination is expected to take well over a year to complete and during that time the spread and evolution of the virus will likely persist $[1,2]$. Furthermore, as vaccine prevalence rises, so does the selective pressure for vaccine resistance. This, together with a sustained large influx of infections over a prolonged period, implies a risk that vaccine-escaping variants will emerge and spread across the population, potentially undermining our current gains [3-6].

This risk of vaccine escape, which exists in any vaccination campaign, is particularly exacerbated by the unique circumstances of COVID-19 [6-16]. Indeed, what keeps the pandemic under control at present and prevents it from proliferating are severe social distancing measures, whether imposed by the authorities or driven by individuals' independent response to the spread. The result, as the short history of this pandemic proves, is an ongoing adaptive behavior that tends to react to the severity of the spread, thus maintaining the effective reproduction number $\mathrm{R}$ at around unity (Figure 1) [17]. Thus, social distancing measures are gradually relaxed as vaccination progresses and population immunity increases. Such a combination of vaccination and adaptive social distancing is unique to the current pandemic, and may have crucial implications for vaccine escape.

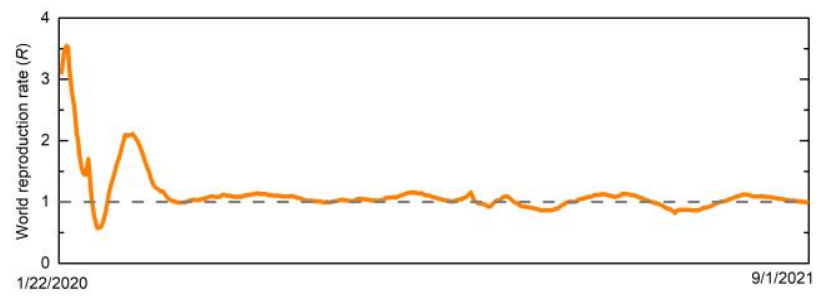

Figure 1: The global reproduction rate (R) during the period $2 / 20$ to $8 / 21[18,19]$. As a result of adaptive social distancing, society converges to a state in which $\mathrm{R}$ is maintained in the vicinity of unity (dashed line). It can therefore be expected that as vaccination progresses and population immunity is gradually acquired, social distancing practices will be relaxed, thus maintaining $R$ at about 1 .

To understand this more clearly, consider the gradual buildup of population immunity as vaccination gains prevalence. Instead of consistently pushing $R$ to below 1 , the increase in population immunity will likely be offset by a relaxation of social distancing, keeping $\mathrm{R}$ around 1 and maintaining a significant rate of infection, a rate that will likely persist until vaccination prevalence approaches herd immunity levels [18]. Indeed, such a pattern has already been observed in countries that have achieved mass vaccination, such as the UK and the US (Figure 2).
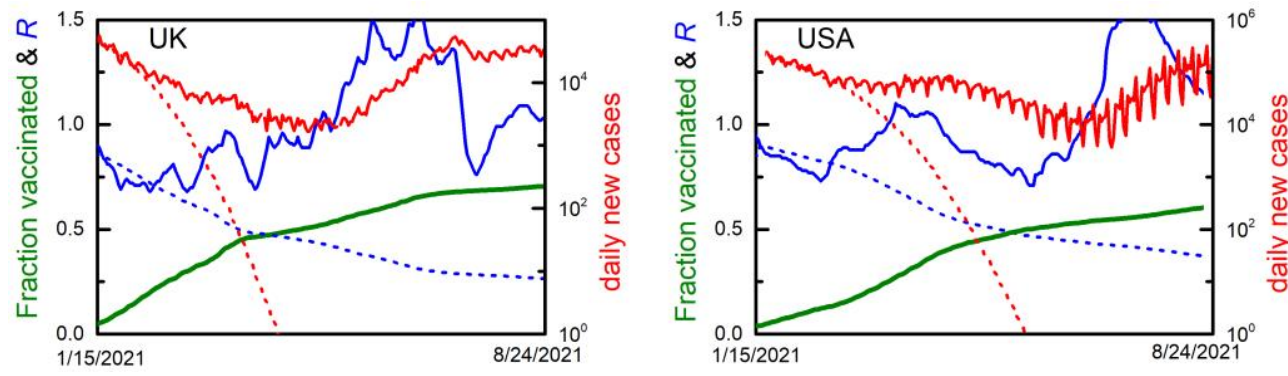

Fraction vaccinated

- $R$ (data)

....R (counterfactual)

- Daily cases (data)

.... Daily cases (counterfactual)

Figure 2: Actual vs. predicted pandemic status in the UK and US during the vaccination campaign. As the fraction of the (first-dose) vaccinated (green line) increases, the extrapolated $R$ (dotted blue line) declines. This extrapolation assumes that factors such as the social distancing restrictions, variant composition, weather, etc. remain unchanged from the start of the vaccination campaign. The empirically measured R (solid blue line) has remained in the vicinity of unity (with a temporary jump to 1.5 just after the introduction of the more infectious delta variant). Furthermore, the extrapolated number of infections (dotted red line) declines much faster than the actual number (solid red line). These trends indeed confirm that adaptive social behavior leads to a relaxation in prophylactic measures, in response to the accumulation of population immunity. 
These unique conditions create a potentially fertile breeding ground for vaccine escape [20]. Once a significant share of the population is vaccinated, a vaccine-resistant variant, which can potentially infect anyone, whether vaccinated or not, has a selective advantage relative to the wild-type strain, as the latter can only infect unvaccinated individuals. Since the wild type's $\mathrm{R}$ is maintained around 1 , this relative advantage translates into an absolute positive growth rate of $R>1$ for the resistant variant, allowing it - if it occurs by a random mutation - to quickly spread throughout the population. This, together with the large number of infections expected during the slow vaccination process, might create a high probability that a mutation will occur and take over the population. Such a mechanism for vaccine escape is, indeed, unique to the current situation in which mitigation involves both vaccination and social distancing, with the latter being relaxed in response to the progress of the former [17, 18, 20,21].

The straightforward solution is to avoid the extended period in which high vaccination prevalence coexists alongside a high rate of infection. Ideally, this would dictate a policy to vaccinate the entire population within a short period of time. Such a solution, however, ignores the main bottleneck to vaccine rollout, namely the inherent limitations on vaccination capacity. To overcome this obstacle, we propose a spatial vaccination strategy which will be shown to dramatically reduce the risk of vaccine escape, even under the existing constraints on the vaccination rate.

The proposed spatial strategy takes advantage of the geographic segregation that often characterizes the population distribution, and the fact that people mainly interact within the region they reside in. We propose to divide each country (or possibly a smaller geographic unit such as a state) into smaller regions that are sufficiently disconnected in terms of social interactions and then sequentially vaccinate one region at a time, thus concentrating the entire country's vaccination capacity in order to quickly bring that region towards herd immunity. Such partitioning would replace the gradual accumulation of nationwide herd immunity. The obvious advantage is that the rapid achievement of herd immunity in each individual region should avoid the prolonged period of interaction between infected individuals and the vaccinated population. Thus, the dangerous combination of high infection rates (the source of mutations) and high vaccination rates (which provide an advantage to resistant strains) is dramatically reduced. Since the majority of infectious interactions are local in nature [22], namely they occur within a single region; cross-infection between regions is rare. Therefore, vaccinating all regions one by one may be able to facilitate a safe and rapid accumulation of local herd immunity in each region, until it is finally achieved for the entire population. The result will be to reach country-level immunity in roughly the same amount of time, but with a significantly lower risk of an escaping variant. $^{1}$

Other considerations may also be important in devising an effective vaccination strategy, and in particular the prioritization of the vulnerable population. We therefore also examine the application of spatial vaccination only after a uniform vaccination of up to $15 \%$ of the population (i.e. the most vulnerable groups). As we demonstrate, this has limited impact on the outcome of the proposed spatial vaccination strategy. The reason for this is that most of the additional risk of vaccine escape due to uniform vaccination occurs only once the vaccine coverage is well above $15 \%$ - prior to that the resistant variant's selective advantage is small.

Spatial vaccination allows for additional (relatively low-cost) measures that further reduce the risk of vaccine escape and are not applicable or are too costly under a uniform vaccination regime. First, an effort can be made to identify and isolate infections by the resistant variant in the vaccinated areas. Such variant contact tracing is likely to be successful since in vaccinated areas, which are clear of wild-type infections, every short

\footnotetext{
1 The model emphasizes the small-numbers evolutionary dynamics that determine whether a mutation will survive under different vaccination strategies. This key factor yields an opposite conclusion as compared to [15], whereas the mutant with an advantage is assumed to survive for sure.
} 
infection chain is highly likely to originate from the resistant variant. This measure is difficult to apply under the current vaccination regime, in which resistant variant infections may be hidden among the predominant wild-type infections. Second, the authorities can impose limitations on population movement between the vaccinated and unvaccinated regions. Such limitations would not be overly burdensome if the order of vaccination is wisely planned, with the goal of keeping the vaccinated and unvaccinated areas geographically contiguous, with one (moving) border between them. Third, the authorities may impose a short, moving lockdown that is applied in each region during or just prior to vaccination. Such a localized and brief lockdown can be more easily enforced relative to prolonged countrywide lockdowns, which impose a devastating individual and societal burden.

Finally, the spatial strategy is effective not only in mitigating vaccine escape, but also in reducing the overall number of infections. This is because, as vaccination progresses, the infections in regions that reach herd immunity will cease much earlier than under uniform vaccination. In fact, if the number of regions is sufficiently large, the total number of infections is reduced by close to $50 \%$, since the infections in a region will on average end after half of the nationwide vaccination time.

The application of the spatial strategy in the real world requires its adoption by governments who will need to tackle the logistic hurdles of a strategy intended to reduce the probability of an adverse event whose likelihood is unknown at this stage. Moreover, while the emergence of a resistant variant is a global problem, each country has only a small effect on its likelihood and thus has only a small incentive to contribute effort to reducing it. Thus, even if our results are logically and scientifically sound, it is unlikely that a coordinated global effort to apply the spatial strategy would be possible during the current vaccination process. However, in the case of a negative scenario, in which an escape variant does become dominant, the spatial strategy can play a beneficial role in a second round of vaccination with an updated vaccine.

\section{Analysis}

We consider the susceptible-infected-recovered (SIR) model and augment it with vaccination and mutations (Appendix 1) [23, 24]. The population is assumed to adapt its behavior by means of social distancing in order to maintain $\mathrm{R}$ at approximately 1 . As a result, we observe a roughly constant rate of new infections, which we set at $10 \%$ of the population per year. ${ }^{2}$ Following one year of such dynamics, we introduce the vaccine whose rollout and production rates make it possible to vaccinate the population within one additional year. ${ }^{3}$ The vaccinated territory (a country, state or other distinct geographic unit) is divided into $\mathrm{K}$ regions such that, on average, only $\mathrm{C}=1 \%$ of a person's interactions are out-of-region. ${ }^{4}$ We vaccinate all regions sequentially up to $80 \%$ coverage. Hence, a specific region $\mathrm{k}(\mathrm{k}=1, \ldots, \mathrm{K})$ can be in one of three states at any given moment: pending vaccination as it awaits its turn, undergoing vaccination, and post-vaccination, at which point the campaign progresses to vaccinating region $\mathrm{k}+1$. Regions that are pending vaccination continue to accumulate infections at the constant rate of $10 \% / y e a r$. Similarly, regions undergoing vaccination also experience a constant $10 \%$ annual infection rate until they reach herd immunity. This is a consequence of their adaptive social distancing,

\footnotetext{
2 This rate represents a reasonable global 4:1 infection-to-case ratio (the total number of world cases in the last 12 months is about 170 million within a population of 7.8 billion). Changing the rate of infection (as long as it is not too high) has almost no effect on our comparative results. More precisely, if we take an infection rate of $5 \%$ and double the mutation probability, then the escape probabilities under all of the various regimes are almost identical.

${ }^{3}$ For simplicity, we consider one-dose vaccination and immediate full immunity following vaccination.

${ }^{4}$ We can imagine a world made up of many countries, each divided into $\mathrm{K}$ regions, such that in step $\mathrm{k}$ each country vaccinates the population in its own region $\mathrm{k}$. In terms of the model, world region $\mathrm{k}$ is the union of all region $\mathrm{k}$ 's worldwide.
} 
which is relaxed as immunity accumulates $[17,18]$. After herd immunity is surpassed, infections quickly decrease and social distancing ceases.

Mutations occur with a small probability, denoted by $\mu$, at each infection event. Hence, an individual carrying the wild type may infect a susceptible individual who may then, with probability $\mu$, acquire a vaccine-resistant strain. ${ }^{5}$ If such a mutant occurs, we model the process of subsequent infection using a discrete random walk process until it either dies out or takes over (see Appendix 1). For expositional simplicity, we assume for now that the resistant strain (1) has the same basic reproduction number as the wild-type strain, (2) is fully resistant to the vaccine, and (3) cannot infect those who have recovered from prior infection with the wild type. These assumptions are relaxed in Section 5.

\section{Illustrative example}

To demonstrate the merits of the spatial vaccination strategy, in Figure 3 we illustrate the different vaccination scenarios for $\mathrm{K}=3$ regions over a one-year vaccination phase. First, we examine simultaneous vaccination, in which all three regions are treated concurrently (Figure 3: panels a-c). The vaccine rollout (purple line) occurs at the same pace in all three regions over the course of the year. During this period, as the population adapts its behavior to maintain $\mathrm{R}$ at around 1 , we continue to observe a roughly constant stream of infection (red line) in all regions. This roughly stable level declines sharply once the herd immunity threshold is crossed (the dashed grey line). (Note that the different variables have different scales and are presented together in order to show the interplay between them.)

Sporadic instances of the resistant strain (the black lines) occur at random locations/points in time, denoted by 1,2,3 and 4 . For example, instance 1 occurs in Region 1 one month after the start of the vaccination campaign ( $t=1)$. At that time, the vaccine prevalence is still low. The resistant mutant thus has no significant selective advantage and therefore fails to proliferate. A similar pattern is observed in instance 3 , which occurs at $t=3$ months in Region 2 . However, as the vaccination rollout progresses, the effective reproduction number of the resistant variant increases. Thus, the risk that a mutant will survive and proliferate, indicated by the shaded background, increases from low (light shading) to high (dark shading). This risk then rapidly drops again after herd immunity is surpassed and the infection streams cease. Indeed, instances 2 and 4, which occur at $\mathrm{t}=9$ months and $\mathrm{t}=7$ months, respectively, both have a significant selective advantage and are hence able to spread (steep ascent of black lines). These mutants eventually reach a number of infections beyond which it becomes inevitable that they will take over the population. As a result, we witness vaccine escape, and the simultaneous vaccination campaign fails.

We next consider the same scenario, except that instead of vaccinating the entire population over the course of one year, we sequentially vaccinate the regions in three rounds, with each campaign lasting four months (Figure 2: panels $d$-f). The two escaping variants in instances 2 and 4 are now averted. Instance 2 does not occur since at $\mathrm{t}=9$ months Region 1 is already cleared of the virus. Instance 4 , on the other hand, also occurs in this scenario. However, it fails to proliferate since at the time of its appearance, i.e. at $\mathrm{t}=7$ months, Region 4 has not yet begun to vaccinate, and therefore, in contrast to the previous scenario, this mutant has a low reproduction number (light rather than dark shading), and poses little risk of escape.

Hence, by splitting the yearly nationwide vaccination cycle into shorter regional ones, we significantly reduce the risk of vaccine escape, thus replacing the extended high-risk time window of potential vaccine escape (Figure 2a-c) with a sequence of narrow time windows, one in each region (Figure $2 \mathrm{~d}-\mathrm{f}$ ).

\footnotetext{
${ }^{5}$ This simplistic model of resistant variant emergence ignores various biological facts, which are discussed in detail in Section 6.
} 
Region 1

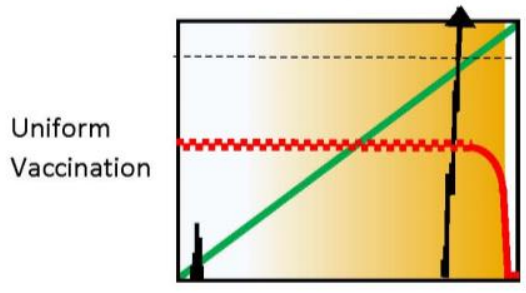

Mutant instance: 1

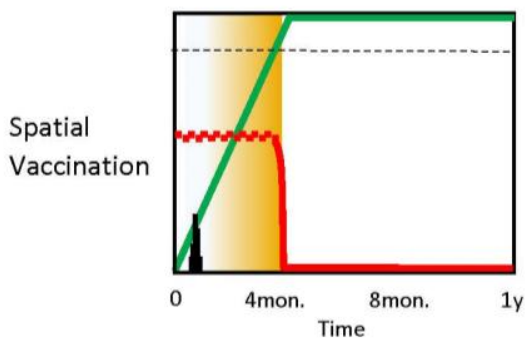

Region 2

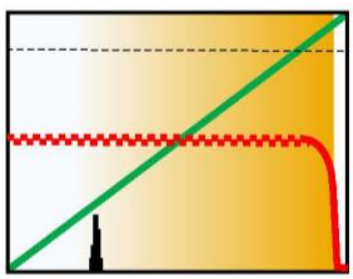

3

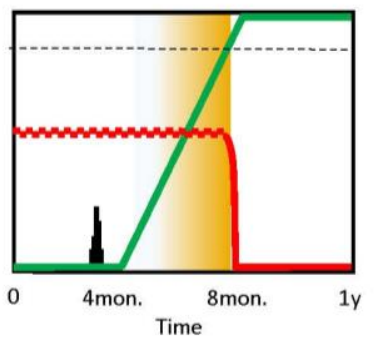

Region 3

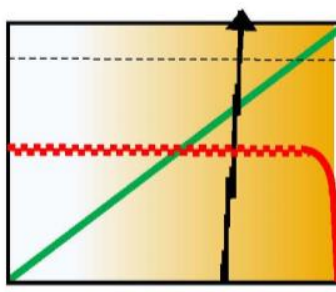

4

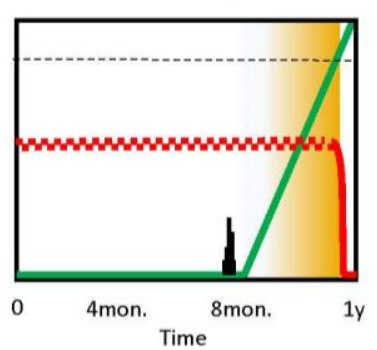

- Proportion vaccinated

--.. Herd Immunity level

Wild-type infections (Large numbers)

- Mutant's initial infections (Small numbers)

Darker shade $=$ Higher risk for mutant takeover (Large mutant's R + many wild-type infections)

Figure 3: Illustration of uniform vaccination (top panels) vs. spatial vaccination (bottom panels) with 3 regions. As the proportion of the vaccinated population (green line) increases from 0 to 1 , social distancing measures are gradually relaxed. Thus, the resistant variant's $R$ increases and with it the chance that a mutation will survive and become dominant (degree of orange shading). The large number of wild-type infections (red line) remains roughly constant until vaccination crosses the herd immunity level (dashed line). At that point, infections quickly decline and the risk of escape (orange shading) diminishes. Four potential instances of resistant mutants (1-4, black lines) are considered and the random walks of the (very small) number of infections in their early stages are illustrated. Under uniform vaccination (top panels), instances 2 and 4 occur in an environment with a large $R$ (dark shading) and succeed in proliferating to numbers from which takeover is almost guaranteed. Under spatial vaccination (bottom panels), in instance 4 the mutation encounters a low $R$ and dies out; mutation 2 does not even occur since in region 1 the stream of wild-type infections ends early on due to the rapid vaccination campaign. Instances 1 and 3 occur, under both scenarios, in environments with a small $\mathrm{R}$ and therefore die out.

\section{Simulation results}

We now turn to computing the probability of vaccine escape. The risk of such an event depends, first and foremost, on the probability of a resistant mutation $\mu$. It is worth emphasizing that vaccine escape is a global problem, since a mutation in any country, even outside the territory currently being vaccinated, will eventually reach all countries and potentially undermine the vaccination campaign globally. Therefore, even if $\mu$ is extremely small, given the large-scale transmission of infection worldwide, the risk is not negligible. To account for this in our analysis, we focus not only on instances of mutation within the population of our simulated territory, but rather on all potential mutations on a global scale, i.e. among a population of $N=$ $7.8 \times 10^{9}$. The challenge is that the value of $\mu$ is unknown. Thus, while the probability of a single point mutation in SARS-COV-2 can be computed from its genetic properties, the probability that a combination of mutations will generate a variant resistant to the current vaccines cannot be calculated. We thus consider a broad range of mutation probabilities, spanning three orders of magnitude, from extremely rare $\left(\mu=10^{-10}\right)$ to highly frequent $\left(\mu=10^{-7}\right)$. Under a mutation rate of $\mu<10^{-10}\left(<N^{-1}\right)$, it is unlikely that even one mutation will occur, even in the case that $10 \%$ of the world population is infected over the course of one year, and therefore escape is very unlikely. With a rate of $\mu>10^{-7}$ mutants are present at any given moment among the estimated $10^{7}$ infected individuals in each infection cycle, and therefore an escape is almost certain even under instantaneous vaccination. For each value of $\mu$ within the range of interest, namely $10^{-10}<\mu<10^{-7}$, we simulated 50000 realizations and calculated the escape probability $P$ from the number of realizations in which a resistant strain was able to propagate and take over the population. ${ }^{6}$

\footnotetext{
${ }^{6}$ Additional parameter values for the simulation are: $R_{0}=4$; infection cycle of 4 days.
} 
The results are presented in Figure 4. We first consider the ideal scenario in which the entire population is vaccinated instantaneously, i.e. in a single day (the black curve). Under these conditions, the only risk of vaccine escape originates from mutations that occurred prior to the vaccination campaign. We find that for $\mu>10^{-7}$ escape is practically unavoidable $(P=1)$. This marks the upper bound on $\mu$, beyond which we cannot hope to avert vaccine escape. Of course, instantaneous vaccination is unattainable in practice. Therefore, the black curve in Figure 4 represents our ideal benchmark to which we will compare the different strategies.

We next consider simultaneous vaccination over the course of one year (the red curve). As expected, we find that the extended vaccination period exhibits a higher probability of escape. Specifically, we observe a window of $10^{-7}>\mu>10^{-10}$ in which the year-long vaccination campaign leads to significant additional risk.

Finally, we consider our proposed spatial vaccination strategy, which divides the vaccinated territory into $\mathrm{K}=10$ regions, each with $\sim 10 \%$ of the population (the dark blue curve). The results clearly show that spatial vaccination eliminates a large part of the excess risk, bringing us closer to the desired benchmark. For $\mathrm{K}=100$ (light blue curve), we only observe a marginal additional benefit, which is an indication of the bounds on the potential benefit of spatial vaccination.

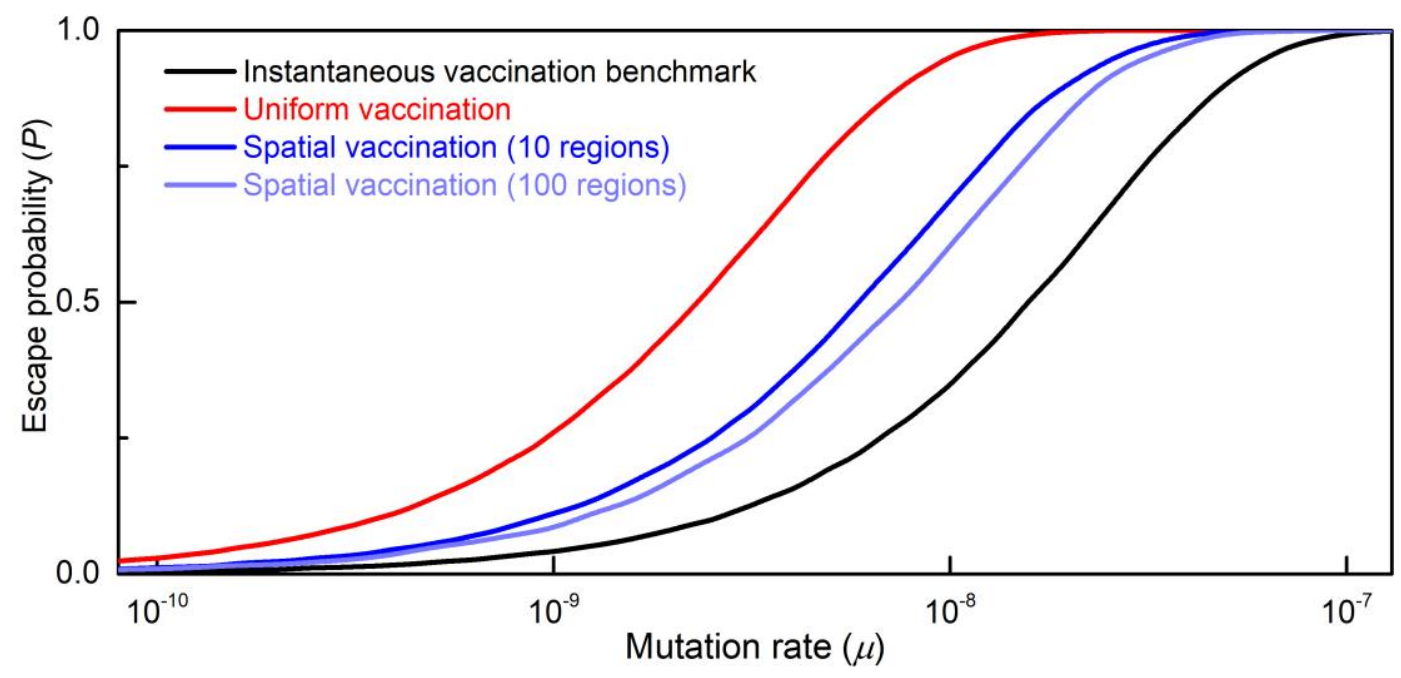

FIGURE 4: Probability of vaccine escape as a function of the mutation rate under various vaccination regimes. For mutation rates between $10^{-10}$ and $10^{-7}$, the probability of escape under the current regime of uniform one-year vaccination (red curve) is far higher than under the benchmark of instantaneous world vaccination (black curve). Spatial vaccination with $\mathrm{K}=10$ regions (dark blue curve) subject to the same one-year constraint restores about $50 \%$ of the excess risk. Increasing the number of regions to $\mathrm{K}=100$ (light blue curve) generates a modest additional gain.

\section{Quantifying the differences in escape risk}

Obtaining a single number that measures the differences in escape risk under the various vaccination regimes is not straightforward. For example, the difference in escape probability is large for intermediate mutation rates but small for very low or very high ones. Using the ratio of escape probabilities does not solve the problem either since again its value is not constant, becoming smaller at high mutation rates. We therefore propose a more suitable metric for comparing vaccination regimes: the difference in the respective mutation rates that lead to the same escape probability under the two regimes. In Appendix B, we formally prove that this measure is constant across different escape probabilities. Thus, the curves in Figure 4 are lateral shifts of one another, and one number, i.e. the horizontal distance, suffices to compare two regimes. 
With this metric in hand, we can quantify the results of the simulation above. The excess risk due to a uniform one-year vaccination regime relative to the instantaneous vaccination benchmark is 0.8 orders of magnitudes. That is, uniform vaccination increases the escape risk to the same extent as in the case that mutations were $10^{0.8}=6.3$ times more frequent. Spatial vaccination with $\mathrm{K}=10$ regions restores 0.42 orders of magnitudes or in other words about $50 \%$ of the excess risk. Thus, moving from uniform to spatial vaccination allows for a mutation rate that is $10^{0.42}=2.6$ times higher for the same escape risk.

The importance of reducing the risk by 0.4 orders of magnitudes depends on our assumption regarding the probability distribution of $\mu$. We discuss ways to address this issue in Section 6 . Note also that this result hinges on the simplistic assumption that resistant variants are as infectious as the wild type, which was made for purposes of simplifying the exposition. In section 5 , we make a more realistic assumption that the escape variant has a lower basic reproductive number than the wild type in view of the fact that immune evasion may require deleterious mutation in the virus $[25,26]$. Under such assumptions, we obtain an even stronger advantage of spatial over uniform vaccination. Moreover, we show in Section 4 that using spatial vaccination makes it possible to employ a number of complementary measures that are infeasible under uniform vaccination. These measures improve the effectiveness of the spatial strategy to an even greater extent.

\section{Reducing the total number of infections}

Apart from reducing the risk of vaccine escape, spatial vaccination has a second advantage: a dramatic reduction in the number of infections with the wild-type strain. This is because in vaccinated regions which reach herd immunity, the stream of infections ceases much earlier than under uniform vaccination. As a rough approximation, if the number of regions is sufficiently large, the total number of infections is reduced by close to $50 \%$ as compared to the uniform vaccination regime. To see this, note that region $\mathrm{k}=1 \ldots \mathrm{K}$ experiences infection from time 0 until time $\mathrm{k} / \mathrm{K}$ and thus, for the average region infections end at time $(1+\mathrm{K}) / \mathrm{K}$, which approaches $1 / 2$ for large $K$. Thus, for the average region, infections last for half a year rather than one year, as under uniform vaccination. ${ }^{7}$

\section{The partition into regions}

Setting $\mathrm{K}$, the level of spatial partitioning, involves a crucial tradeoff. On the one hand, the larger $\mathrm{K}$ is, the smaller each region will be and hence the faster it will be vaccinated. As a result, we limit the time window in which the vaccinated and infected individuals interact and in that way suppress the escape risk for each region. This would dictate dividing the territory into as many regions as possible. On the other hand, if the regions are overly small they may not be socially separable. For example, if we try to partition a city or a densely populated country, individuals from different regions will likely interact, thus undermining the benefit of the spatial strategy. We therefore seek an optimal balance between the number of regions $\mathrm{K}$ and the level of interregional coupling, $\mathrm{C}$.

\footnotetext{
${ }^{7}$ This back-of-the-envelope calculation assumes that the threshold at which we end vaccinating one region and move to the next is fixed and is equal to that reached after one year under uniform vaccination. In fact, there is an interesting tradeoff between minimizing infections and minimizing the probability of a resistant variant, with the vaccination threshold lower for the former strategy. This is because the tail of infections that occur after the herd immunity level has been reached, and when the variant has a high $\mathrm{R}$, poses an increased risk of vaccine evasion. To reduce this risk, it is important to continue vaccination in order to achieve a steep decline in infections down to zero. In contrast, if the main purpose is to reduce the total number of infections, then it is better to move to the next region earlier on, allowing the infection stream to diminish more quickly.
} 
To systematically examine this tradeoff, in Figure 5 a we present the escape probability $P$ vs. the number of regions $\mathrm{K}$, under various values of the coupling parameter $\mathrm{C}$. We first observe that the benefit from increasing $K$ reaches saturation for $K>20$, which is in line with our previous results in Figure 4 , which showed only a marginal gain from increasing $\mathrm{K}$ from 10 to 100. As expected, we also find that as $\mathrm{C}$ increases the effectiveness of the spatial strategy declines. Specifically, for $C>5 \%$ the spatial vaccination strategy ceases to offer a significant benefit.

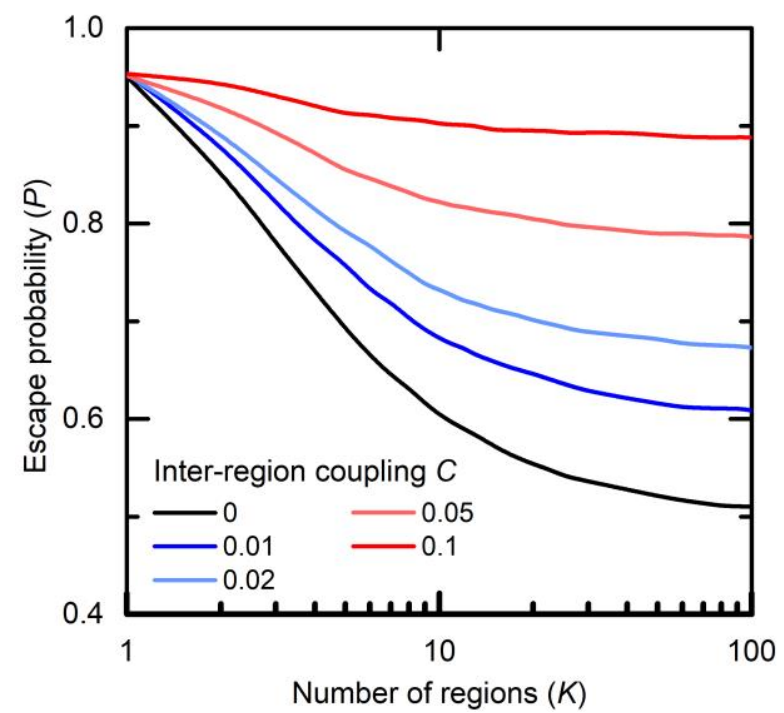

Figure 5: The number of regions vs. the separation between regions (for $\mu=10^{-8}$ ): In the case of a contact ratio of $1 \%$ (blue line) or $2 \%$ (light blue line) we observe a steep reduction in the probability of escape as the number of regions $\mathrm{K}$ increases up to about 20 ; the benefit of increasing $\mathrm{K}$ further is limited (the curves almost flatten out). With a contact ratio of $5 \%$ (orange) or $10 \%$ (red) the benefit of increasing $\mathrm{K}$ is small, so that $\mathrm{C}=5 \%$ and $\mathrm{K}=10$ is equivalent to $\mathrm{C}=1 \%$ and $\mathrm{K}=3$. The black line indicates the ideal limit at which there are no contacts between regions $(\mathrm{C}=0)$.

This makes it possible to establish guidelines for spatial partitioning. In each country or equivalent territory, we should seek to establish naturally separated regions to whatever extent possible, so as to achieve a separation of $C \cong 1 \%$, namely that in any region only $1 \%$ of interactions between people are out-of-region. Therefore, partitioning a city or large metropolitan area, for example, is likely to be inefficient, while separating along county, province or state lines is more likely to achieve the desired effect.

It is important to note that even if a considerable percentage of the population lives in a large metropolitan area that cannot be divided for purposes of partial vaccination, it is still beneficial to divide the remaining population according to region. This is because the escape risk from each region is cumulative. That is, the factor that determines the probability of a resistant variant takeover is the proportion of infections (i.e. mutation opportunities) that will occur while there is a highly vaccinated population in that region (resulting in a high reproduction rate for resistant variants). Thus, for example, if $30 \%$ of the population lives in a metropolitan area that takes three months to vaccinate, and the remaining $70 \%$ can be divided into 12 regions that take one month each to vaccinate, then $70 \%$ of the $\mathrm{K}=1$ probability is reduced by the factor that $\mathrm{K}=12$ generates, and $30 \%$ is reduced by the factor that $\mathrm{K}=4$ generates.

\section{The role of inter-region coupling and the order of vaccination}

To better understand the role of inter-region coupling $\mathrm{C}$, consider a region that has already been vaccinated. Having reached local herd immunity, infections in that region have ceased and hence, given adaptive social behavior, practically all restrictions have been lifted. Due to the vaccination, the wild-type's reproduction number is kept below one, and thus any wild-type infection that arrives from another region dies out quickly. However, if the resistant strain arrives, then, absent social distancing, it benefits from a very high $\mathrm{R}$ (which 
equals $R_{0}$ ) and therefore quickly ignites a new breakout of infection. Note that for any region, this risk only exists post-vaccination since prior to vaccination social distancing restrictions limit the ability of a mutant to take off, and incoming infections are negligible relative to the flow of within-region infections.

The implication is that interactions between vaccinated regions or between unvaccinated regions do not pose a problem. What matters is the separation, at each point in time, between the vaccinated regions and the not-yet-vaccinated regions. Thus, the partitioning into regions and the order of vaccination should be designed such that bordering regions, which are likely to have a high level of contact, are vaccinated adjacently in time, so that there is one "moving" border between the vaccinated and unvaccinated regions. Therefore, $\mathrm{C}$ should be interpreted as the ratio of an average person's interactions with people on the other side of the "border" to his interactions with people on his own side of the border. Under this interpretation, $C \sim 1 \%$ or lower appears to be reasonable.

\section{Complementary measures}

The spatial vaccination strategy makes it possible to employ a few simple and relatively low-cost complementary measures that reduce the risk of vaccine escape even further, including travel limitations between vaccinated and unvaccinated regions, contact tracing for resistant variants and temporary regional lockdowns during vaccination. Importantly, these measures are not applicable or are too costly under a uniform vaccination regime. We assess the effectiveness of each of these measures by repeating the simulation carried out in Section 2 with the necessary modifications (see Appendix 1 for a formal treatment).

\section{a. Limitations on travel between vaccinated and unvaccinated regions}

The authorities can impose temporary limitations on travel between vaccinated and not-as-yet vaccinated regions. This will avoid the potential spillover of mutations from unvaccinated to vaccinated regions. In the latter restrictions are lifted and any entering mutant will enjoy a large reproduction number and a high likelihood of success. Indeed, the coupling between same-type regions has only little effect and thus the effect of reducing travel just between vaccinated and not-as-yet vaccinated regions is almost the same as that of a reducing travel between all the regions (i.e., reducing the coupling parameter $\mathrm{C}$ ). Importantly, if the vaccinated regions as a whole and the unvaccinated regions as a whole are each kept contiguous, with one moving border between them as explained above, then the limitations on travel - only across that border - will not be overly burdensome.

Figure 6a presents the effect of travel limitations between vaccinated and unvaccinated regions. The red and black lines represent, as they did in Figure 4 above, the escape probabilities under uniform vaccination and instantaneous vaccination, respectively. We observe that the probability of escape under spatial vaccination with $\mathrm{K}=10$ regions and $\mathrm{c}=1 \%$ (blue curved) is reduced when travel is restricted by a factor of 5 (light blue curve). We also consider the case of $\mathrm{C}=5 \%$ (brown curve). We see that restricting travel between vaccinated and unvaccinated regions by a factor of 5 (light brown) is almost as effective as having $\mathrm{C}=1 \%$ initially (blue curve).

\section{b. Contact tracing of the resistant variant}

Recall that, in vaccinated regions, the wild-type strain has been eradicated and moreover, any wild-type infection imported from other regions dies quickly due to the high vaccination level. Therefore, any infection chain among vaccinated individuals - even if short - is highly suspected of belonging to the resistant strain. As a result, contact tracing targeted specifically at resistant variants will be highly effective. It is worth emphasizing that variant contact tracing can be effective only under the spatial vaccination strategy. Indeed, under uniform vaccination resistant-variant infections are hidden among the many wild-type infections, and hence contact tracing becomes infeasible. 
We repeat the simulation under the assumption that contact tracing of infections with the resistant strain can be effectively applied only when - due to vaccination - the stream of new wild-type infections in region $\mathrm{k}$ falls to below $1 / 10$ of the pre-vaccination level and that when contact tracing is implemented it reduces the resistant variant's reproduction number by a factor of 2 . The outcome is depicted by the green line in Figure $6 b$.

\section{c. A moving temporary lockdown}

A third measure that can further reduce the escape risk under spatial vaccination is the application of a shortterm, moving lockdown of the region currently being vaccinated. Indeed, a month-long lockdown will be more acceptable to the population than the year-long lockdown required to achieve the same objective under uniform vaccination, and it has a much better cost-benefit ratio. Figure $6 \mathrm{~b}$ (purple line) presents the outcome under stricter temporary restrictions, only in the region being vaccinated, such that the reproduction number there is further reduced to 0.8 (rather than 1 ).

Note that an alternative scenario which leads to the same outcome is a delay in the behavioral response to the increased level of vaccination. Since the increasing beneficial effect of vaccination is counteracted by a delayed relaxation of social distancing, the region being vaccinated will enjoy a reproduction number of less than 1.
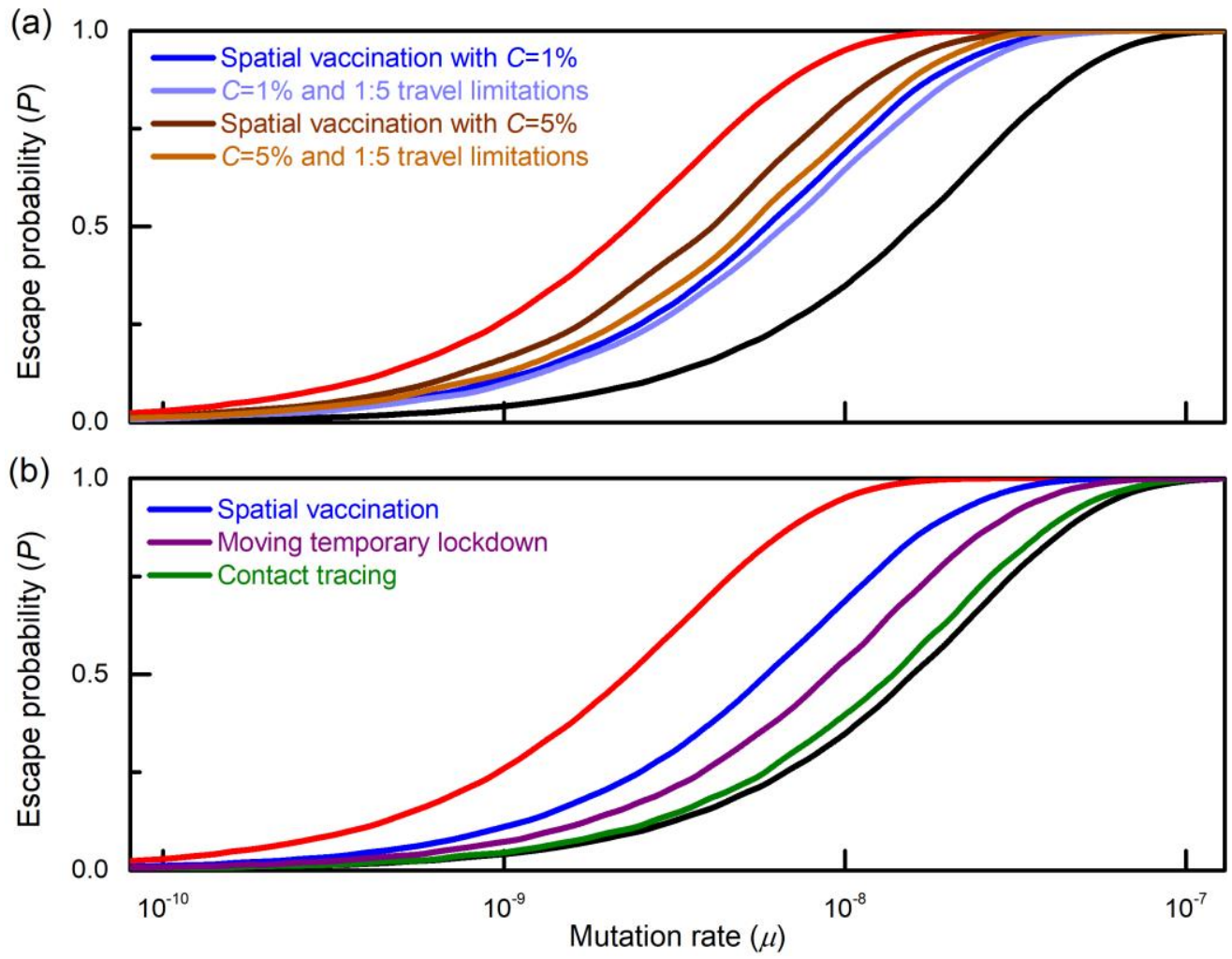

Figure 6: Complementary measures. (a) Restricting travel between vaccinated and unvaccinated regions by a factor of 5 reduces the probability of escape (light brown vs. dark brown and light blue vs. blue curves). In particular, if the initial inter-region coupling is large ( $\mathrm{C}=5 \%$, dark brown), the outcome under travel restrictions (light brown) is almost as good as in the case of $\mathrm{C}=1 \%$ (blue), i.e. implying that travel between same-type regions has little effect. (b) A short temporary lockdown of the region being currently vaccinated, that reduces the effective reproduction number there to 0.8 (rather than 1 ) reduces the escape probability (purple vs. blue curves). Contact tracing for the resistant variant - which is assumed to be feasible only in vaccinated regions that are almost clear from wild-type infections, and in that case reduces the variant's effective reproduction number $\mathrm{R}$ by a factor of 2 - has a dramatic effect on the escape probability (green vs. blue curves). 


\section{Extensions}

To simplify the exposition, we have so far assumed that an "ideal" escape variant, which (1) has the same basic reproduction number as the wild type, (2) cannot infect people with antibodies from a prior wild-type infection (i.e. we assumed two-way cross immunity) and (3) is fully resistant to the vaccine. In reality, there are a large number of conceivable combinations of mutations, in which each of the above assumptions may be violated. In this section, we examine the consequences of relaxing these assumptions. We also consider a modification of the spatial strategy that prioritizes the vaccination of the more vulnerable population in all regions before switching to sequentially vaccinating the different regions.

\section{a. Escape is costly}

It is reasonable to assume that a vaccine-resistant variant will be deficient relative to the wild type with respect to its ability to infect unvaccinated individuals. This follows from our definition of the wild type, as the dominant variant prior to vaccination, i.e. that with the maximal $R_{0}$ vs. unvaccinated among all variants. The resistant variant, which is selected from a strict subset of the variants - those resistant to the vaccine, will typically have a lower $R_{0}$ vs. unvaccinated. Moreover, vaccines such as Moderna's and Pfizer's SARS Cov2 vaccines are designed to target a conserved protein, such as the spike protein. This design is based on the rationale that targeting conserved and highly essential viral proteins is likely to impose a high cost on immuneevading mutations [27]. (Mutant binding data of the type produced in [28, 29] may be used to assess the existence and sign of potential correlation between, for example, antibody and receptor binding.)

We capture this idea using the deficiency ratio, denoted by $d$, which is the ratio between the reproduction number of the mutant strain and that of the wild type, both in a naive population. Hence, $d=1$ implies no deficiency, which is what has been assumed up to this point, while $d=0.5$, for example, means that the variant's reproduction number is half that of the wild type. In Figure $7(a, b)$ we present the probability of vaccine escape vs. the variant's deficiency ratio $d$ and the mutation rate $\mu$, under simultaneous vaccination (7a) and spatial $\mathrm{K}=10$ vaccination (7b). For completeness we consider the range $0.4 \leq d \leq 1.2$; however, as argued above, the middle of this range should be seen as more probable. As expected, in both cases we observe low risk (blue) in the bottom-right corner, either because mutations are rare (small $\mu$ ) or because they are deleterious to the virus (small $d$ ). In the opposite corner, where $\mu$ and $d$ are large, we observe a high risk (red) under both regimes. The two areas are separated by a band of intermediate risk (green). The crucial point is that under spatial vaccination the low-risk blue area is expanded, while the high-risk red area contracts to only the extreme upper-left corner. This clearly shows that spatial vaccination consistently mitigates the risk of vaccine escape throughout the entire range.

Importantly, it can be seen that the benefit of spatial vaccination, namely how far it shifts the green and red areas to the right, is greater when the variant has higher deficiency (smaller $\mathrm{d}$ ): while for $d=1$ the rightward shift is about 0.42 orders of magnitude $(\times 2.6)$ of $\mu$, for $d=0.8$ it is about 0.56 orders of magnitude $(\times 3.6)$ of $\mu$ (these are the respective horizontal distances in between the dotted and solid black curves in Figure $7 \mathrm{~b}$ ). The reason for the difference lies in the variant's ability to survive before vaccination gives it an advantage. With $d<<1$, the variant has an $R<<1$ before vaccination and in its early stages. Thus, if it appears in these

\footnotetext{
${ }^{8}$ The fact that some of the existing variants (such as Beta) show some degree of vaccine escape together with a higher reproduction number does not imply that a typical escape variant would not have a fitness deficiency. These variants, which emerged during a period with no vaccination and hence no fitness advantage for vaccine resistance, have proliferated because they improve upon the previous dominant strain with respect to their infectiousness parameters (an improvement that is expected as part of the virus's adaptation process to the new human host). In other words, their proliferation is not due to their improved ability to escape the vaccine. For a very helpful review of SARS-CoV-2 variants and immune escape see [6].
} 
early stages then it will likely not survive. Only variants born late in the vaccination process, when their $\mathrm{R}$ exceeds unity, are able to survive, implying that spatial vaccination has a major advantage due to the shortening of the high-risk period. With $d=1$, in contrast, variants born in the earlier stages have a higher chance of survival - under any vaccination regime - thus reducing the relative advantage of spatial vaccination.

\section{b. A variant that also infects the recovered}

We next examine the effect of the variant's ability to also infect individuals with antibodies from prior infection with the wild type. As expected, the results, for both $K=1$ (Figure $7 \mathrm{c}$ ) and $\mathrm{K}=10$ (Figure 7d), indicate that such a scenario increases the risk of escape relative to the case in which the variant cannot infect the recovered (Figures $7 \mathrm{a}$ and $7 \mathrm{~b}$ ). Note however that even under this scenario, in which the variant also infects the recovered, the spatial vaccination continues to maintain a clear advantage, as can be seen in the enlarged low-risk area (blue) and the diminished high-risk area (red).

\section{c. Escape is partial}

Another important issue we examine is partial vaccine escape, i.e. the vaccine provides some protection against the escape variant. This scenario appears to fit our current experience with some of the SARS-CoV-2 variants $[30,31] .^{9}$

To quantify this scenario, we introduce a parameter measuring the severity of vaccine escape, denoted by $e$. Setting $e=1$ represents full escape, which is the scenario considered up to this point; $e=0.5$, for example, means that the vaccine is partially effective against the variant so that the variant escapes it with only $50 \%$ probability. In Figure 7(e,f), we set $d=0.7$ and examine the impact of introducing $e<1$. It can be seen that the contour lines now have a steep, almost vertical slope in both heat maps. Hence, the risk is largely independent of $e$ within the range $0.4 \leq e \leq 1$. Importantly, this risk continues to fall as we shift from simultaneous vaccination $(K=1)$ to spatial vaccination $(K=10)$.

\section{d. Vaccinating the vulnerable population first}

An important consideration in the allocation of vaccinating capacity is whether to prioritize the vulnerable population, such as the elderly. We thus consider a mixed vaccination regime, in which uniform vaccination of a certain fraction of the population (the vulnerable) is first implemented across all regions, followed by spatial vaccination of the remaining population.

Figure 7h presents the heat maps for this scenario in which there is uniform vaccination of $15 \%$ of the population (Figure $7 \mathrm{~g}$ presents the fully uniform vaccination case for comparison). We see that spatial vaccination remains almost as effective as in the case where the vulnerable population was not prioritized (Figure $7 \mathrm{~b}$ ), with the equi-probability lines shifting to the right by $0.17-0.52$ orders of magnitude (7h) instead of $0.25-0.56$ orders of magnitude as in Figure $7 \mathrm{~b}$. Therefore, the spatial strategy appears to be robust to prioritizing the vulnerable population.

The reason that the uniform vaccination of $15 \%$ of the population has no detrimental effect on the spatial vaccination's success is that most of the additional risk of vaccine escape due to uniform vaccination occurs only once the vaccine coverage is well above 15\%; prior to that the resistant variant has little selective advantage, if any. ${ }^{10}$

\footnotetext{
${ }^{9}$ Note, however, that as of now the existing variants have emerged in countries that have not yet achieved high levels of vaccination. As vaccination progresses, we might encounter variants that are able to better escape the vaccines. ${ }^{10}$ While in our model all individuals have the same number of interactions, in reality the more vulnerable population tends to have fewer interactions. Thus, a population fraction of $15 \%$ in our model corresponds to $20-30 \%$ in reality.
} 

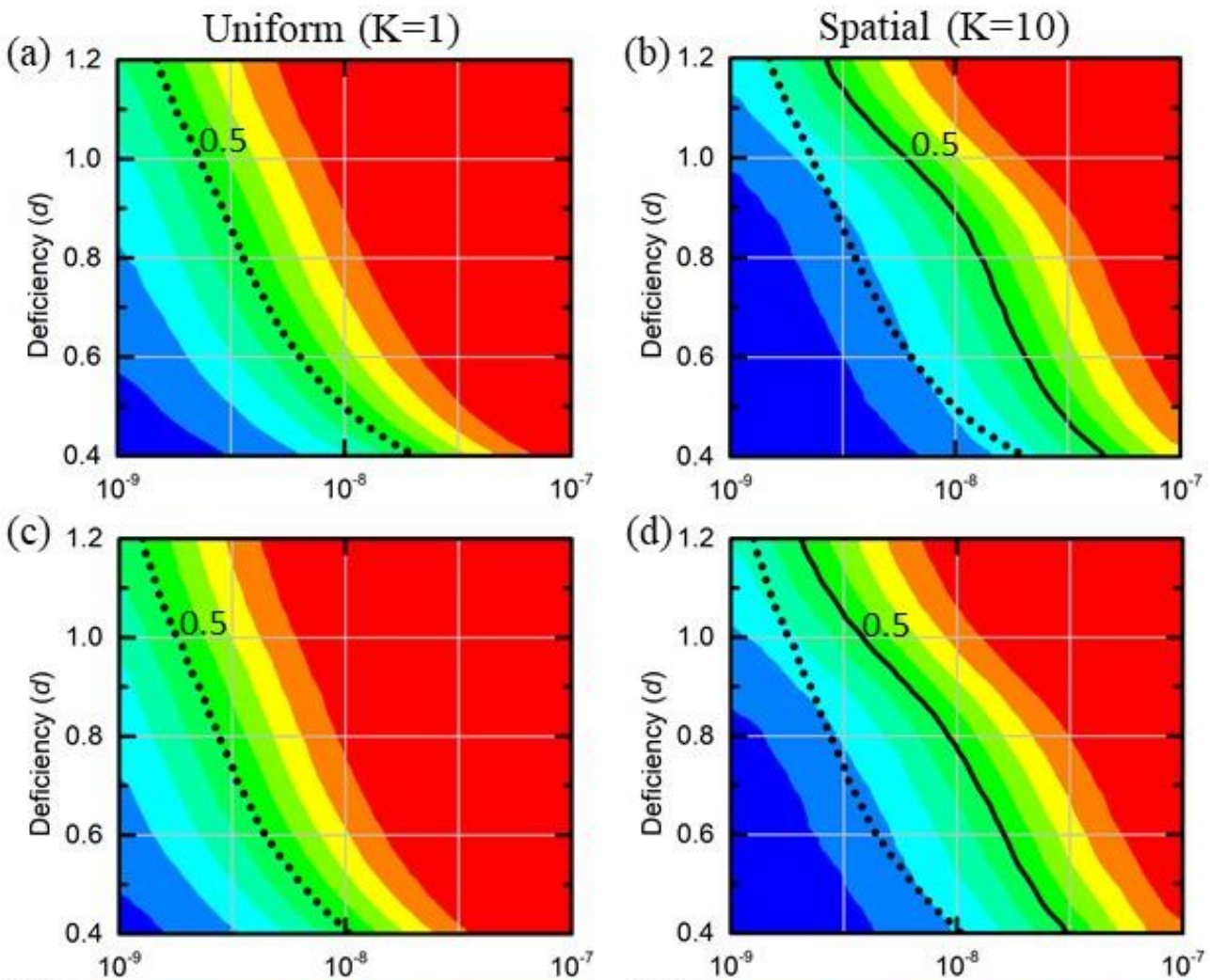

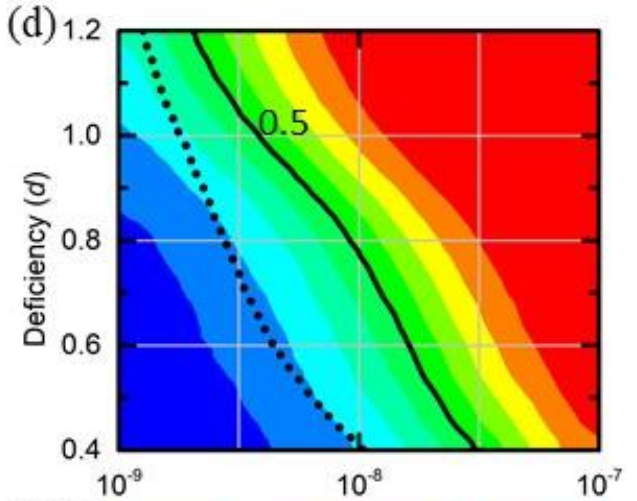

\section{Escape probability}

$(P)$

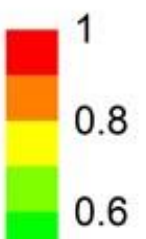

(f) 1.0
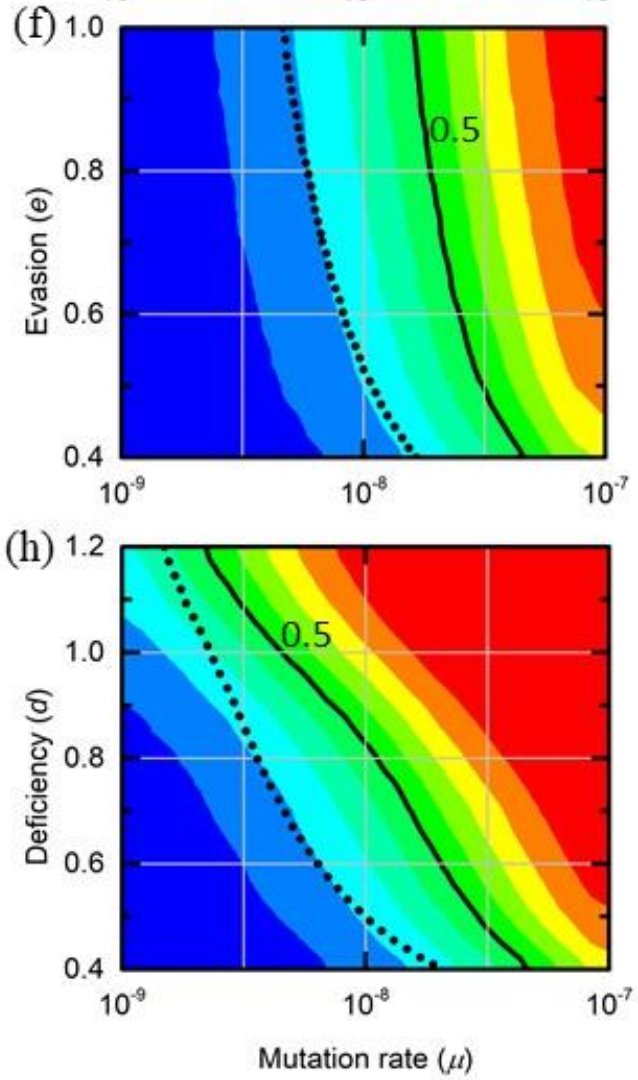

Figure 7: Probability of escape for different mutation rates under spatial uniform vaccination (left) vs. spatial vaccination (right)

$(a, b)$ As a function of the variant's deficiency ratio $d$ relative to the wild-type strain.

$(c, d)$ Same as $(a, b)$ except that the variant can infect individuals who have recovered from infection with the wild type.

$(\mathrm{e}, \mathrm{f})$ As a function of the variant's escape capability $e$.

(h) Spatial vaccination $(K=10)$ following uniform vaccination of $15 \%$ of the population (the more vulnerable) as compared to $100 \%$ uniform vaccination $(\mathrm{g})$. 


\section{Concluding remarks}

- A uniform global SIR model. The simplified SIR model we present assumes a homogeneous world in which social distancing maintains the reproduction number $R$ at unity and the stream of infections is identical in all countries and regions. Of course, the real world is characterized by a high degree of heterogeneity even across proximate regions $[18,19]$. Nonetheless, we believe that our qualitative results still hold. The key idea is that under the current slow and uniform vaccination regime, and in view of the adaptive nature of social distancing measures, the world is going to experience continued high infection rates for an extended period of time. A significant proportion of these infections will occur when vaccination levels are high, implying a high reproduction rate and therefore a high probability of survival for an escape variant. Our analysis, which "mistakenly" uses the average R in each of the regions, ignores the nonlinearity of the survival probability in R. ${ }^{11}$ However, this effect is small and more importantly, the effectiveness of spatial vaccination - which in each locality dramatically shortens the time during which a high infection level and a high R coexist - remains.

- The mutation process. Our model treats the complex process of mutation in a highly simplified manner, assuming that upon each instance of infection, the virus mutates with some probability. In reality, a complex mutation that enables vaccine escape does not take place at the time of infection, but rather it is the result of a combination of single-point mutations occuring within the host, as the virus reproduces within his body. However, this internal process is of little relevance to our analysis since - apart from very rare cases - infections almost always involve a single strain randomly drawn from the cloud of mutations within the infector. ${ }^{12}$ Hence, for simplicity we assume that with probability $\mu$, a specific host acquires a vaccine-resistant mutation, which is then passed on to other individuals via further infection. We model this hidden process as if the virus mutates upon infection. Our model, however, ignores the possibility of a drift by which a sequence of single-point mutations occurs during the transmissions between individuals, such that only the sum of these mutations generates vaccine escape.

- The range of the mutation rate and the significance of the reduction in escape risk. While the probability of a single-point mutation in the case of the SARS-COV-2 virus can be computed from its genomic properties, the probability that a combination of mutations generates a variant that is resistant to the current vaccines is unknown at this stage. On the optimistic side, none of the currently prevailing variants fully escapes the current vaccines [32,33], and it appears that most of them are simply improvements of the virus that are to be expected in its adaptation process in the human host. On the pessimistic side, this can be explained by the fact that mass vaccination, which leads to selective pressure for vaccine resistance, has taken place until recently only in countries that account for a small proportion of the world's population [37,38].

In Section 2 we showed that the type of vaccination regime matters when the mutation rate $\mu$ is within the range of $10^{-10}$ to $10^{-7}$. That is, within that range there is a gap between the best-case instantaneous vaccination benchmark and the worst-case slow vaccination regime that has actually been adopted.

\footnotetext{
${ }^{11}$ A mutant's survival probability is approximately $1 /(1+1 / R)$ for sufficiently large $R$.

12 The characteristics of the internal process are more important in studying drug resistance, as in [34, 35], or in studying partial (one-dose) vaccination which may generate an inter-host fitness advantage for a resistant variant together with a continued increased viral load [36].
} 
We have shown that spatial vaccination eliminates $50 \%$ of the excess risk (and even more with the help of the complementary measures described in Section 4).

Another way to assess the benefit of spatial vaccination is to consider the metric introduced in Section 2 , according to which spatial vaccination permits a higher mutation rate for the same escape risk - by about 0.5 orders of magnitude on average (across possible values of $d$ ) before taking into account complementary measures. Translating this number into a probability that escape will be avoided depends on our prior on $\mu$, namely what range of values do we view as being reasonable. Lobinska et al. [38] provides a methodology to compute an upper bound based on the fact that a vaccine-resistant variant has not appeared until now in the highly vaccinated countries (which currently account for 5$10 \%$ of world population) and arrives at an estimate of about $10^{-6}$. However, without a lower bound it is hard to justify employing the spatial strategy. Such a lower bound will be generated if we encounter the adverse scenario that a resistant variant emerges during the first round of global vaccination (and the lower bound will be stricter the earlier the variant is encountered). If this leads to a second round of vaccination with an updated vaccine, then the spatial strategy might be considered. Moreover, the combination of the lower and upper bounds may provide us with a fairly narrow range, which will justify the use of spatial vaccination.

- Might spatial vaccination slow the vaccination process? There are two kinds of limitations on the ability to concentrate effort in small regions in order to accelerate vaccination: vaccine production capacity and logistic capabilities. The former is in fact a global constraint and therefore there is no difficulty in directing global output to specific regions. This constraint does not therefore limit the effectiveness of the spatial strategy. The second constraint - which is binding - is determined by how easily medical personnel can be moved from one region to another, or the availability of facilities in which vaccination can take place. Thus, the overall pace of vaccination might be slowed by adopting the spatial strategy, and this should be taken into account. However, the experience with COVID-19 shows that although vaccination is slow at first, the pace improves quickly over time. It therefore might even turn out to be more efficient to create the necessary logistics infrastructure and move it from region to region, thereby saving on start-up time. 


\section{References:}

[1] Different COVID-19 Vaccines | CDC. Available at: https://www.cdc.gov/coronavirus/2019ncov/vaccines/different-vaccines.html.

[2] Safe COVID-19 vaccines for Europeans | European Commission. Available at:

https://ec.europa.eu/info/live-work-travel-eu/coronavirus-response/safe-covid-19-vaccines-europeans en. (Accessed: 19th March 2021)

[3] N. G. Davies, et al., Estimated transmissibility and severity of novel SARS-CoV-2 Variant of Concern 202012/01 in England. medRxiv preprint doi: https://doi.org/10.1101/2020.12.24.20248822 (2020).

[4] H. Tegally, et al., Sixteen novel lineages of SARS-CoV-2 in South Africa, Nat. Med. 27, 440-446 (2021)

[5] S. Cele, et al., Escape of SARS-CoV-2 501Y. V2 variants from neutralization by convalescent plasma, Nature 593, 142-146 (2021).

[6] W. T. Harvey, et. al, SARS-CoV-2 variants, spike mutations and immune escape, Nature Reviews Microbiology, 19, 409-424 (2021).

[7] H. Tegally, et al., Emergence and rapid spread of a new severe acute respiratory syndrome-related coronavirus 2 (SARS-CoV-2) lineage with multiple spike mutations in South Africa. medRxiv preprint doi: https://doi.org/10.1101/2020.12.21.20248640 (2020).

[8] A. Rambaut, et al. Preliminary genomic characterisation of an emergent SARS-CoV-2 lineage in the UK defined by a novel set of spike mutations - SARS-CoV-2 coronavirus / nCoV-2019 Genomic Epidemiology Virological. (2020). Available at: https://virological.org/t/preliminary-genomic-characterisation-of-anemergent-sars-cov-2-lineage-in-the-uk-defined-by-a-novel-set-of-spike-mutations/563.

[9] P. Wang, et al. Increased Resistance of SARS-CoV-2 Variants B.1.351 and B.1.1.7 to Antibody Neutralization. bioRxiv Prepr. Serv. Biol. doi:10.1101/2021.01.25.428137 (2021).

[10] L. L. Luchsinger and C. D. Hillyer, Vaccine efficacy probable against COVID-19 variants. Science 371, 1116-1116 (2021).

[11] T. N. Starr, et al. Prospective mapping of viral mutations that escape antibodies used to treat COVID-19. Science 371, 850-854 (2021).

[12] T. N. Starr, et al. Deep Mutational Scanning of SARS-CoV-2 Receptor Binding Domain Reveals Constraints on Folding and ACE2 Binding. Cell 182, 1295-1310.e20 (2020).

[13] A. J. Greaney, et al. Complete Mapping of Mutations to the SARS-CoV-2 Spike Receptor-Binding Domain that Escape Antibody Recognition. Cell Host Microbe 29, 44-57.e9 (2021).

[14] R. N. Thompson, E. M. Hill and J. R. Gog, SARS-CoV-2 incidence and vaccine escape. Lancet Infect. Dis. 21, 913-914 (2021).[15] P. J. Gerrish, et al. How unequal vaccine distribution promotes the evolution of vaccine escape. medRxiv preprint doi: https://doi.org/10.1101/2021.03.27.21254453 (2021).

[16] S. Cobey, D. B. Larremore, Y. H. Grad, and M. Lipsitch, Concerns about SARS-CoV-2 evolution should not hold back efforts to expand vaccination. Nature Reviews Immunology 21, 330-335 (2021)

[17] J. S. Weitz, S. W. Park, C. Eksin, and J. Dushoff, Awareness-driven behavior changes can shift the shape of epidemics away from peaks and toward plateaus, shoulders, and oscillations, PNAS 2020117 (51) 3276432771 (2020) 
[18] F. Arroyo-Marioli, F. Bullano, S. Kucinskas and C. Rondón-Moreno, Tracking R of COVID-19: A new realtime estimation using the Kalman filter. PloS One 16.1: e0244474 (2021).

[19] Our world in data, https://ourworldindata.org/explorers/coronavirus-data-explorer

[20] K. Linka, M. Peirlinck, and E. Kuhl, The reproduction number of COVID-19 and its correlation with public health interventions, medRxiv preprint doi: 10.1101/2020.05.01.20088047 (2020)

[21] T. C. Williams, T. C. and W. A. Burgers, W. A. SARS-CoV-2 evolution and vaccines: cause for concern? Lancet Respir Med 9, P333-335 (2021)

[22] N. Schläpfer, M., Dong, L., O’Keeffe, K. et al., The universal visitation law of human mobility, Nature 593, 522-527 (2021).

[23] T. H. Luong, Mathematical Modeling of Vaccinations: Modified SIR Model, Vaccination Effects, and Herd Immunity. (Portland State University, 2019). doi:10.15760/honors.712.

[24] A. Huppert and G. Katriel, Mathematical modelling and prediction in infectious disease epidemiology. Clinical Microbiology and Infection 19 999-1005 (2013)

[25] A. H. Melnyk, A. Wong and R. Kassen, The fitness costs of antibiotic resistance mutations, Evol Appl. 8(3): 273-283 (2015).

[26] Q. Li, et. al., SARS-CoV-2 501Y.V2 variants lack higher infectivity but do have immune escape, Cell 184, Pages 2362-2371.e9 (2021)

[27] K. A. Swanson, et. al., A respiratory syncytial virus (RSV) F protein nanoparticle vaccine focuses antibody responses to a conserved neutralization domain, Science Immunology 5, 47 (2020).

[28] A. J. Greaney, et. al., Comprehensive mapping of mutations in the SARS-CoV-2 receptor-binding domain that affect recognition by polyclonal human plasma antibodies, Cell Host \& Microbe 29, 463-476 (2021).

[29] T. N. Starr, et. al. Deep Mutational Scanning of SARS-CoV-2 Receptor Binding Domain Reveals Constraints on Folding and ACE2 Binding, Cell 5, 1295-1310.e20 (2020)

[30] S. Nasreen, et. al., Effectiveness of COVID-19 vaccines against variants of concern, Canada, medRxiv preprint doi: https://doi.org/10.1101/2021.06.28.21259420 (2021)

[31] Public Health England, https://www.gov.uk/government/news/vaccines-highly-effective-against-b-1617-2-variant-after-2-doses

[32] S. A. Rella, Y, A. Kulikova, E. T. Dermitzakis and F. A. Kondrashov, SARS-CoV-2 transmission, vaccination rate and the fate of resistant strains, medRxiv preprint doi: https://doi.org/10.1101/2021.02.08.21251383 (2021)

[33] D. A. Kennedy and A. F. Read, Why does drug resistance readily evolve but vaccine resistance does not?, Proc. R. Soc. B 284: 20162562 (2017)

[34] Y. Iwasa, F. Michor and M. A. Nowak, Evolutionary dynamics of escape from biomedical intervention, Proceedings of the Royal Society of London. Series B: Biological Sciences 270.1533: 2573-2578 (2003).

[35] R.K. Plowright, et. al., Reproduction and nutritional stress are risk factors for Hendra virus infection in little red flying foxes (Pteropus scapulatus), Proceedings of the Royal Society B: Biological Sciences 275.1636: 861-869 (2008). 
[36] C. M. Saad-Roy, S. E. Morris, C. J. E. Metcalf, M. J. Mina, R. E. Baker, J. Farrar, E. C. Holmes, O. G. Pybus, A. L. Graham, S. A. Levin, B. T. Grenfell, C. E. Wagner, Epidemiological and evolutionary considerations of SARS-CoV-2 vaccine dosing regimes. Science 372, 363-370 (2021).

[37] Harvey, W.T., Carabelli, A.M., Jackson, B. et al. SARS-CoV-2 variants, spike mutations and immune escape. Nat Rev Microbiol 19, 409-424 (2021).

[38] M. A. Nowak, G. Lobinska, A. Pauzner, A. Traulsen and Y. Pilpel, Evolution of resistance to COVID-19 vaccination with dynamic lockdown, Research Square preprint doi: https://doi.org/10.21203/rs.3.rs$\underline{646895 / v 1}$

\section{ACKNOWLEDGEMENTS}

We thank Jonathan Gershoni and Ron Milo for helpful comments and discussions.

\section{FUNDING}

X.Z. was supported by the NSF of China under Grant No. 12105117 and by the Fundamental Research Funds for the Central Universities (Grant No. 21621007).

\section{AUTHOR CONTRIBUTIONS}

All authors designed and did the research; X.Z. analyzed the data and conducted the numerical simulations; The project was conceived and led by A.P., following an idea of Y.Pauzner; A.P. and B.B. were the lead writers of the paper.

\section{CONFLICTING INTERESTS}

None

CODE AND DATA AVAILABILITY

Available upon request. 


\section{Supplementary Materials:}

\section{Appendix A: The Model}

A world with population $N$ is naturally divided into $K$ identical regions $k=1 \ldots K$ with little interaction between them. Let $0<c<<1$ (the contact ratio) denote the proportion of a person's interactions that are with people living outside of her own region. The dynamics of the wild-type infection in each region follows a deterministic SIR model, with interactions between the regions. We set the recovery rate $r$ to 1 so that time is measured in infection cycles (about 4 days in the COVID-19 case) and for simplicity set the death rate to 0. The wild type's basic reproduction rate $R_{0}$ is set to 4 , which is about that of the current dominant strains, so that the infection rate is $\beta_{0}=r R_{0}=4$. We assume that social distancing measures - multiplicative factors $l_{k}$ - are set such that the rate of infection in each region does not exceed an acceptable proportion $h$ of the population (per 4-day infection cycle).

We augment the model with a vaccination process $v_{k}(t)$ that starts at time $t_{0}$ (= one year after the pandemic's start) and is subject to an aggregate capacity constraint $\Sigma v_{k}(t) \leq v$ such that $80 \%$ of the population can be vaccinated within one year (i.e., $v=80 \% \cdot 4 / 365$ is the proportion vaccinated per infection cycle). Both the susceptible and the recovered are vaccinated. Under uniform vaccination, $v_{k}(t)=v$, i.e. a proportion $v$ of every region's population is vaccinated per infection cycle. Under spatial vaccination, $v_{k}(t)=v \cdot K$ for $t_{k-1} \leq$ $t \leq t_{k}$, where $t_{k}$ is the point in time at which $80 \%$ of region $k^{\prime}$ s population is vaccinated; otherwise $v_{k}(t)=$ 0 . Thus, when it is region $k^{\prime}$ s turn, it is vaccinated at a $K$-fold faster rate than under uniform vaccination. Under "mixed" vaccination, we start at time $t_{0}$ with the uniform regime until $15 \%$ of the population in each region is vaccinated and then switch to the spatial regime.

Each wild-type infection has a probability $\mu$ of turning into a vaccine-resistant variant ("mutant"). While the wild type cannot infect vaccinated individuals, the mutant can. However, it suffers a fitness deficiency such that its basic reproduction number $R_{0}^{m}$ equals $d \cdot R_{0}$ (while we typically expect $d$ to be lower than 1 , we also analyze the case in which it is above 1 ). If a new mutant occurs, we track the (discrete) number of infections using a random walk process until it either dies out or succeeds in generating a large number of infections (and then becomes dominant). The parameter $e \leq 1$ captures the degree of the variant's escape from the vaccine ( 1 means full escape; 0 means no escape). We also consider two cases: that the resistant variant can or cannot infect individuals who have recovered.

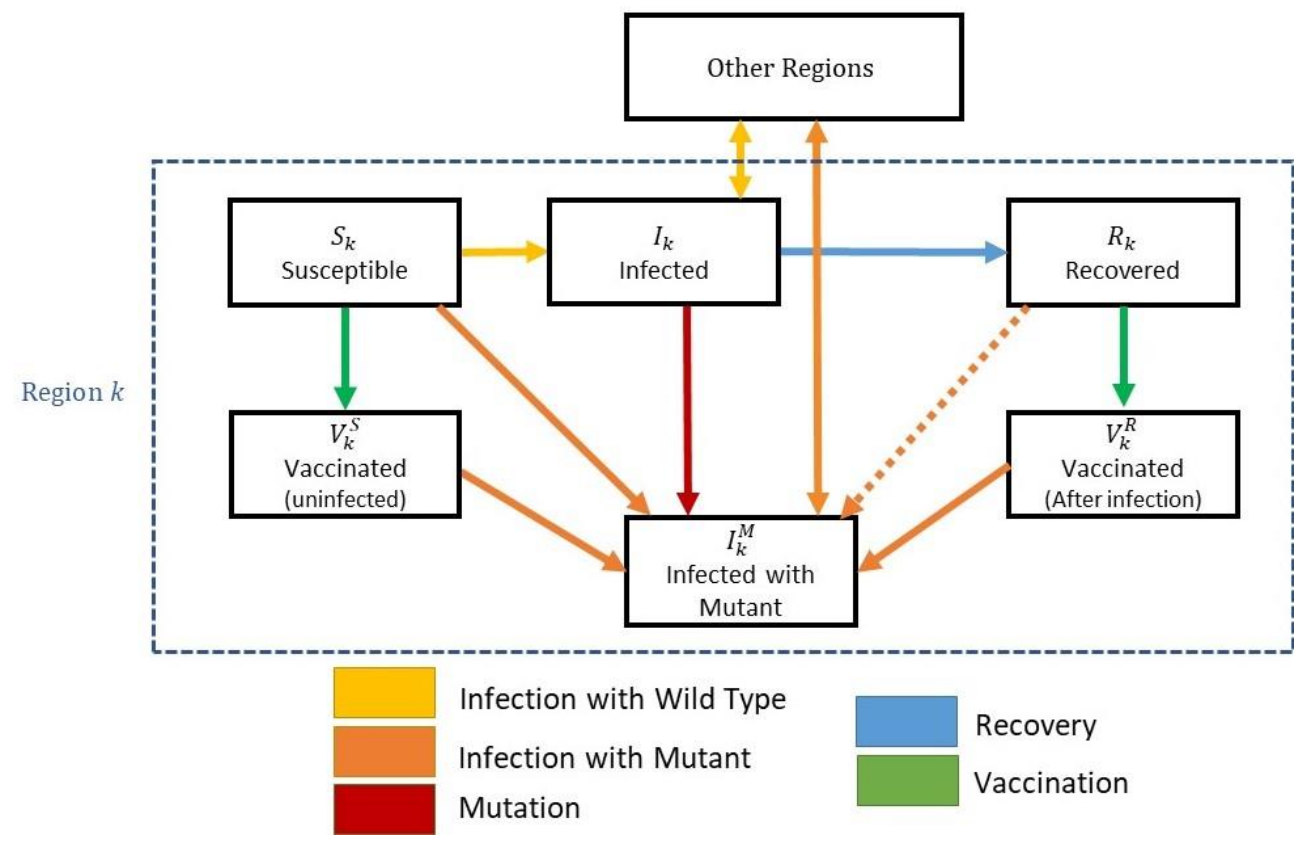


The deterministic SIR equations for the wild-type strain in each region $k$ are as follows (all state variables are defined as proportions of the population, i.e. between 0 and 1 ):

W1.

$$
\begin{aligned}
& \text { Infected: } \quad \dot{\mathrm{I}}_{k}=l_{k} \beta_{0} S_{k} \hat{I}_{k}(1-\mu)-r I_{k} \\
& \text { where } \hat{I}_{k}=(1-c) I_{k}+\frac{c}{K-1} \Sigma_{j \neq k} I_{j} \text { is the effective infection pool for region } k \\
& \text { and where } l_{k}=\min \left(1, \frac{h}{\beta_{0} S_{k} \hat{I}_{k}(1-\mu)}\right) \text { so that } \dot{\mathrm{I}}_{k}=\min \left(\beta_{0} S_{k} \hat{I}_{k}(1-\mu), h\right)-r I_{k}
\end{aligned}
$$

W2. Susceptible: $\dot{S}_{k}=-l_{k} \beta_{0} S_{k} \hat{I}_{k}(1-\mu)-v_{k} \frac{S_{k}}{S_{k}+R_{k}}$

W3. Recovered: $\dot{\mathrm{R}}_{k}=r I_{k}-v_{k} \frac{S_{k}}{S_{k}+R_{k}}$

W4. Vaccinated (and were not infected earlier) ${ }^{13}: \dot{V}_{k}^{S}=v_{k} \frac{S_{k}}{S_{k}+R_{k}}$

W5. Vaccinated (and were infected and recovered earlier): $\dot{V}_{k}^{R}=v_{k} \frac{R_{k}}{S_{k}+R_{k}}$

The initial conditions are: $I_{k}=h, S_{k}=1-h, R_{k}=V_{k}^{S}=V_{k}^{R}=0$.

The process for the resistant strain is discrete. Initially, the (absolute) number $I_{k}^{m}$ of mutant infections in region $k$ is 0 . Each $I_{k}^{m}$ evolves according to a random walk, dictated by three Poisson processes: (1) arrival of new mutations; (2) infections by the resistant variant; and (3) recoveries. The arrival rate of these processes are given by:

M1. Mutation $\left(I_{k}^{m}\right.$ increased by 1$): \mu \cdot l_{k} \beta_{0} S_{k} \hat{I}_{k} \cdot \frac{N}{K}$

(Note that for not yet vaccinated regions his is simply $\mu \cdot h \cdot \frac{N}{K}$.)

M2. Infections ( $I_{k}^{m}$ increased by 1$)$ :

- Case 1 (variant does not infect the recovered): $l_{k} \beta_{0}^{m}\left(S_{k}+e V_{k}^{S}\right) \widehat{I_{k}^{m}}$

○ Case 2 (variant infects the recovered): $l_{k} \beta_{0}^{m}\left(S_{k}+e\left(V_{k}^{S}+V_{k}^{R}+R_{k}\right)\right) \widehat{I_{k}^{m}}$ where $\beta_{0}^{m}$ equals $d \cdot \beta_{0}$ and where $\widehat{I_{k}^{m}}=(1-c) I_{k}^{m}+\frac{c}{K-1} \sum_{j \neq k} I_{j}^{m}$ is the effective infection pool for region $k$.

(For simplicity we assume that the variant cannot infect people currently infected by the wild type.)

M3. Recoveries $\left(I_{k}^{m}\right.$ decreased by 1$): r \cdot I_{k}^{m}$

\section{Outcome of the simulation (one iteration)}

- We say that the variant has taken over when $\Sigma_{k} I_{k}^{m}$ grows to beyond $30 .{ }^{14}$

- We say that the pandemic is over and the variant has died out when $\Sigma_{k} I_{k}<1 / N$ and $\Sigma_{k} I_{k}^{m}=0$.

\footnotetext{
${ }^{13}$ The separation between the two types of vaccinated individuals is needed only for the case in which the resistant variant cannot infect people who have already recovered from the wild type (see Case 1 below). In this case it can infect only those in $\mathrm{V}^{\mathrm{S}}$. If the variant is not resistant to wild-type antibodies, i.e. it also infects the recovered (Case 2), then we can merge $\mathrm{V}^{S}$ and $\mathrm{V}^{\mathrm{R}}$ into one group $\mathrm{V}$.

${ }^{14}$ Since mutations are rare, infections can reach 30 only if the reproduction number is much larger than 1 . Moreover, since the lockdown is further eased as vaccinations proceed, the mutation's effective reproduction number can only grow. Thus, the path from 30 to taking over is almost guaranteed. Indeed, simulations show that setting a higher threshold does not result in a lower probability of reaching it.
} 
The complementary measures (see Section 4) are modeled using the following modifications:

a. Travel restrictions: Let $c^{-}<c$ denote the reduced interaction rate between regions with different vaccination statuses and denote the region being currently vaccinated by $k^{*}$. We change the definition of $\hat{I}_{k}$ as follows:

$$
\begin{aligned}
& \hat{I}_{k}=\left(1-\frac{\left(k^{*}-2\right) c+\left(K-k^{*}+1\right) c^{-}}{K-1}\right) I_{k}+\frac{c}{K-1} \sum_{j<k^{*}, j \neq k} I_{j}+\frac{c^{-}}{K-1} \sum_{j \geq k^{*}} I_{j} \text { if } k<k^{*} \\
& \hat{I}_{k}=\left(1-c^{-}\right) I_{k}+\frac{c^{-}}{K-1} \sum_{j \neq k} I_{j} \\
& \hat{I}_{k}=\left(1-\frac{k^{*} c^{-}+\left(K-k^{*}-1\right) c}{K-1}\right) I_{k}+\frac{c^{-}}{K-1} \sum_{j \leq k^{*}} I_{j}+\frac{c}{K-1} \sum_{j>k^{*}, j \neq k} I_{j} \quad \text { if } k>k^{*}
\end{aligned}
$$

and correspondingly the definition of $\widehat{I_{k}^{m}}$.

b. Contact tracing for the resistant strain: In regions that have already been vaccinated and in which the total number of wild-type infections has been significantly reduced, i.e. $\dot{I}_{k}<0.1 h$, we further multiply the resistant strain's infection rates (cases 1 and 2 in $\mathrm{M} 2$ above) by $l^{C T}=0.5$.

c. A moving temporary lockdown: For the region $k^{*}$ being vaccinated, we replace the definition of $l_{k}$ (W1 above) with $l_{k^{*}}=\min \left(0.8,0.8 \frac{1}{\beta_{0} S_{k}}\right)$ which implies that the reproduction number is pushed down to 0.8 rather than to 1 . 


\section{Appendix B: Proof that the metric for escape risk differences (Section 2) is well-defined}

Given a configuration $c$ (vaccination regime and model parameters except for $\mu$ ), denote the stream of new infections at time $t$ by $x_{k}(t, c)$ and the probability of takeover by a mutant that occurs at time $t$ (and assuming that no other mutant occurs any time) by $p_{k}(t, c)$. The probability of escape (i.e. at least one mutant occurring and taking over) is:

$$
P(c, \mu)=1-\prod_{t=0}^{T}\left(1-p_{k}(t, c)\right)^{\mu x_{k}(t, c) d t}
$$

(where $\Pi$ here denotes the geometric integral - the continuous version of the usual product sign.)

\section{Claim:}

If $P\left(c_{1}, \mu_{1}\right)=P\left(c_{2}, \mu_{2}\right)$, then $P\left(c_{1}, \alpha \mu_{1}\right)=P\left(c_{2}, \alpha \mu_{2}\right)$

Note: This claim implies that the difference in the $\mu^{\prime} s$ that lead to the same escape risk $P$ under two different vaccination regimes is independent of $P$. Thus, one number can be used to compare one regime to another. This implies that in Figures 4 and 6 the lines are horizontal shifts of one other. In Figure 7, in each pair of panels and for each value of the vertical parameter $d$ or $e$, all the equi-probability lines (the borders between colors) shift by the same amount and therefore it is sufficient to look at the shift of the $P=50 \%$ line.

\section{Proof:}

Taking the natural logarithm of $1-P(c, \mu)$ computed above we obtain:

$$
\ln (1-P(c, \mu))=\mu \int_{t=0}^{T} x_{k}(t, c) \ln \left(1-p_{k}(t, c)\right) d t .
$$

Thus, $P\left(c_{1}, \mu_{1}\right)=P\left(c_{2}, \mu_{2}\right)$ is equivalent to:

$$
\mu_{1} \int_{t=0}^{T} x_{k}\left(t, c_{1}\right) \ln \left(1-p_{k}\left(t, c_{1}\right)\right) d t=\mu_{2} \int_{t=0}^{T} x_{k}\left(t, c_{2}\right) \ln \left(1-p_{k}\left(t, c_{2}\right)\right) d t
$$

while $P\left(c_{1}, \alpha \mu_{1}\right)=P\left(c_{2}, \alpha \mu_{2}\right)$ is equivalent to:

$$
\alpha \mu_{1} \int_{t=0}^{T} x_{k}\left(t, c_{1}\right) \ln \left(1-p_{k}\left(t, c_{1}\right)\right) d t=\alpha \mu_{2} \int_{t=0}^{T} x_{k}\left(t, c_{2}\right) \ln \left(1-p_{k}\left(t, c_{2}\right)\right) d t .
$$

Clearly, the first equation implies the second. QED. 